\title{
EL SEMINARIO: UN MÉTODO DOCENTE INTEGRADOR E INTERDISCIPLINAR PARA LA FORMACIÓN DE PERIODISTAS
}

\author{
Rosa María Arráez-Betancort ${ }^{1}$ : Universidad Europea Miguel de Cervantes. España \\ rarraez@uemc.es
}

Elvira Jensen-Casado: Universidad Europea Miguel de Cervantes. España $\underline{\text { mejensen@uemc.es }}$

Carolina Pascual-Pérez: Universidad Europea Miguel de Cervantes. España cpascual@uemc.es

\section{RESUMEN}

Siguiendo el concepto de enseñanza-aprendizaje del EEES, las guías y programaciones docentes deben diseñarse para conseguir una educación global del alumno. Buscamos relacionar las asignaturas Literatura y medios de comunicación, Inglés II y Redacción para los medios III, no sólo desde la aplicación de metodologías docentes similares sino desde la secuenciación del aprendizaje. En este trabajo proponemos la aplicación en el aula de nuestra investigación en literatura y periodismo desde una metodología interdisciplinar, aprovechando la coincidencia de nuestras asignaturas en una misma materia denominada "Expresión oral y escrita para los medios de comunicación" en la que se comparten competencias, metodologías y resultados de aprendizaje. La innovación se basa en la reflexión y planificación de clases a partir de la investigación en literatura y periodismo, en concreto del periodismo literario entendido como área de especialización temática y ante la localización mínima de materiales sobre este asunto. El encuentro entre disciplinas, fundamental para el avance de la propia ciencia, permite esa educación global del alumno que le ayude a interrelacionar todos los conocimientos adquiridos a su paso por la universidad y a integrarse en el mercado del trabajo.

PALABRAS CLAVE: Interdisciplinar - Periodismo literario - Seminario - Periodismo especializado - Literatura

\footnotetext{
${ }^{1}$ Autor correspondiente

Rosa María Arráez-Betancort: Doctora. Facultad de Ciencias Humanas y de la Información. Universidad Europea Miguel de Cervantes, Valladolid, España.

Correo: rarraez@uemc.es
} 


\title{
THE SEMINAR: AN INTERGRATING AND INTERDISCIPLINARY TEACHING METHOD FOR THE EDUCATIONAL FORMATIONS OF JOURNALISTS
}

\begin{abstract}
Following the teaching-learning concept of the EEES, the guides and teaching programs must be designed to deliver global education to the student. In this paper we propose the application of our research in literature and journalism in the classroom with an interdisciplinary methodology. We want to relate subjects as Literature and Mass Media, English II and Writing for the Media III not only sharing teaching methodological applications but also partaking the learning steps. We all share teaching skills, methodologies and learning results as we have the same educational matter in our subject: "Written and spoken language in the mass media". The innovation is based on the study and planning of the classes starting from research on literature and journalism, more specifically from literary journalism that should be understood as an area of thematic specification. The innovation is also based on the very few materials we can find on this subject. The encounter between the subjects, which is basic for any science, gives the student a global education that will help him to interrelate all his knowledge acquired in the university and a better integration in the labor market.
\end{abstract}

KEY WORDS: Interdisciplinary - Literary journalism - Seminar - Specialized journalism- Literature

\section{INTRODUCCIÓN}

\subsection{Metodología interdisciplinar y docente para periodistas}

Coincidiendo con las indicaciones del EEES, las guías y programaciones docentes han de contribuir a una educación global, a la interrelación por parte del alumno de todos los conocimientos adquiridos durante su vida universitaria para asegurar con éxito su llegada al mercado laboral.

Las denominadas nuevas metodologías docentes, que no son nuevas por originales sino por aparecer reflejadas en la normativa para contribuir a un mayor protagonismo del estudiante en su proceso de aprendizaje con una implicación más activa, permiten 
La llegada del EEES a la titulación de periodismo, tal y como sucede en otras titulaciones, precisa, tomando como referencia a Mario de Miguel Díaz, la implantación de metodologías acordes con un modelo basado en el aprendizaje del alumno frente al anterior centrado en la enseñanza, (Miguel, 2005, p. 11). Abordar el modo de enseñar en la disciplina de periodismo, siguiendo a Sotelo y Marcos, supone considerar los presupuestos del EEES y el papel de los docentes y alumnos frente a esta nueva realidad educativa (Sotelo \& Marcos, 2008, p. 341). Como afirma López Pan, la formación de los periodistas se resumiría en saber comunicar con sus variantes de saber hablar, escribir, expresarse en imágenes, mirar, escuchar, pensar y saber acerca de la condición humana (López Pan, 2007, p. 72-76).

Como indican los especialistas (Martín-Izard, et al., 2008, p. 204), resulta crucial trabajar la docencia de forma cooperativa con el fin de: “buscar fórmulas de trabajo entre profesores de diferentes disciplinas, -en nuestro caso filología y periodismo-, con la intención de generar un equipo que marque la diferencia entre el equipo y el equipo cooperativo". Puesto que se insiste en redefinir la relación entre profesor y alumno, se trataría de "predicar con el ejemplo" porque "el trabajo cooperativo con los alumnos es una de las técnicas propuestas". Estos autores, siguiendo a Putnam (Martín-Izard, et al., 2008, p. 204), plantean la importancia de la generación de confianza entre docentes con un objetivo similar y donde fluya el intercambio de información que fomente la cooperación, así como los compromisos con el grupo y a título individual.

Mario de Miguel Díaz entiende el método docente como aquellas decisiones procedimentales y de recursos necesarios que han de emplearse "en diferentes fases de un plan de acción que, organizados y secuenciados coherentemente con los objetivos pretendidos en cada uno de los momentos del proceso, nos permiten dar una respuesta a la finalidad última de la tarea educativa" (Miguel, 2005, p. 11).

Insiste este autor en que todo método trae consigo una serie de pasos secuenciados temporal y lógicamente que han de justificarse y explicarse de modo racional. El método necesita también de otras justificaciones psicológicas y contextuales. En un primer caso, por la necesidad de su adecuación a los alumnos que aprenden y, en un segundo término, por el papel que desempeña el contexto en las actividades relacionadas con el aprendizaje. Todos estos elementos característicos deben integrarse en el método de enseñanza elegido.

Resulta imprescindible la coherencia de las acciones con los objetivos presentados y debe darse respuesta a las "intenciones explícitas" que son, en definitiva: "las competencias que el estudiante debe adquirir v/o desarrollar en el proceso 
En este sentido, se hace relevante el uso de técnicas didácticas apropiadas con el fin de potenciar "planteamientos metodológicos globalizadores". Coincidimos con Díaz de Miguel, cuando afirma que "la metodología globalizadora es el intento de ofrecer a cada estudiante los materiales de aprendizaje de la forma más similar a como las informaciones le llegan en la vida cotidiana y profesional" (Miguel, 2005, p. 36-37). Es el estudiante el encargado de construir sus significados y de aplicarlos a las situaciones de la vida cotidiana.

Los alumnos son así capaces de resolver problemas, descubrir nuevos aprendizajes, o establecer continuamente vínculos entre contenidos. Se trata de efectuar tareas que ayuden en la adquisición de conocimientos relevantes para el alumno. Con esta apuesta globalizadora, los estudiantes deben realizar actividades de aprendizaje que precisan del encuentro "simultáneo o sucesivo" de contenidos variados, tales como aptitudes específicas, actitudes, valores o normas.

Por lo tanto, estas y otras aportaciones nos invitan a proponer la aplicación de nuestra investigación en literatura y periodismo en las aulas desde una metodología interdisciplinar, aprovechando la coincidencia de nuestras asignaturas en una misma materia denominada "Expresión oral y escrita para los medios de comunicación" en la que se comparten competencias, metodologías y resultados de aprendizaje. Resulta obvio que las asignaturas que conforman esta materia, con sus competencias, métodos $\mathrm{y}$ resultados, tienen una presencia transversal en el grado en periodismo porque cualquier periodista que se precie necesita dominar el lenguaje de los distintos medios periodísticos y sus géneros de información, interpretación y opinión para insertarse laboralmente.

La innovación se basa en la reflexión y planificación de clases sobre un área poco explotada desde el punto de vista de la investigación en literatura y periodismo, en concreto del periodismo literario entendido como área de especialización temática y ante la localización mínima de materiales sobre este asunto. Se trata, por tanto, de aprovechar la relación existente entre las siguientes asignaturas, Literatura y medios de comunicación, Inglés II y Redacción para los medios III, no sólo desde la aplicación de metodologías docentes similares sino desde la secuenciación del aprendizaje.

El objetivo de relacionar las asignaturas no es otro que el de aprender transversalmente, interconectando conocimientos de manera continua puesto que el alumno va a trabajar con métodos similares pero adaptados a sus posibilidades. Acciones de este talante consolidan grupos de profesores que se coordinan, reflexionan y debaten sobre un mismo fin, aplicando un método común basado en 
Los alumnos deben tomar conciencia de la conexión y complementariedad frente a la "compartimentación del conocimiento en áreas independientes entre sí" porque "no favorece el tratamiento interdisciplinar, ni tampoco la perspectiva de educación global que se pretende" (Martín-Izard, et al., 2008, p. 209). También Elena Martí demanda la necesidad de "presentar problemas reales en los que confluyen perspectivas disciplinares complementarias" (Martí, 2003, p. 287), con el objeto de ampliar y mejorar las relaciones entre conocimientos. Compartimentar en exceso no beneficia a los saberes, como ya planteara Ortega y Gasset en los primeros años del siglo XX, y perjudica el avance de la misma ciencia. Evidentemente, los enfoques interdisciplinares cuentan con la ventaja añadida de potenciar los equipos de trabajo, fundamentalmente para la generación de conocimiento profesional y académico. En nuestro caso, parece claro que el alumno percibe la vinculación entre la teoría y la práctica.

En las asignaturas en las que vamos a realizar este trabajo interdisciplinar partimos de unas competencias genéricas comunes que queremos que consiga el alumno y que coinciden con las recomendadas por autores como Carlos Monereo y Juan Ignacio Pozo: capacidad de análisis, síntesis y juicio crítico, capacidad para trabajar en equipo, capacidad para resolver problemas y tomar decisiones, capacidad para desarrollar el pensamiento creativo, capacidad para comunicar imágenes, ideas o símbolos y la capacidad para aplicar los conocimientos a la práctica (Monereo \& Pozo, 2003, p. 15-30). Y también dentro de las competencias específicas de cada asignatura se dan coincidencias evidentes.

Las actividades realizadas en las distintas asignaturas se enfocan de manera que el alumno alcance resultados de aprendizaje, tales como el dominio de la lengua española en los planos fonológico, gramatical y sintáctico, su comprensión correcta de manera escrita, el desarrollo de la expresión analítica y sintética, la emisión razonada de juicios, críticas e ideas creativas, el desarrollo de recursos para exponer en público y de interés por el conocimiento de otras realidades culturales literarias contemporáneas o el trato correcto con las fuentes informativas.

\section{METODOLOGÍA}

La presente investigación se basa en el análisis de las anteriores escuelas de pensamiento que han tratado el tema de la investigación en literatura y periodismo desde una metodología interdisciplinar, aprovechando la coincidencia de nuestras asignaturas en una misma materia denominada. Se trata de analizar los principales medios existentes hoy y aplicarles un método analítico textual que permita producir inferencias fenomenológicas o estructuras de pensamiento novedoso basado en este 


\section{ANÁLISIS Y DISCUSIÓN}

\subsection{Un método docente integrador e interdisciplinar en el caso de la literatura y el periodismo: el seminario}

La puesta en marcha de un grupo de investigación denominado Literatura y Periodismo ha propiciado el estudio del periodismo literario como área temática, frente a la consideración hasta entonces del periodismo literario únicamente como forma de hacer periodismo. Nuestra intención era y es abordar el papel del periodismo en la difusión de la literatura desde los géneros informativos, interpretativos y de opinión, con sus correspondientes variantes periodísticas. Pero también el contribuir a potenciar la cultura, en este caso la lectura literaria. Nuestros análisis periodísticos y literarios han permitido investigar sobre las metodologías científicas del análisis de contenido con la correspondiente elaboración de plantillas enriquecidas desde la filología y el periodismo, siempre a la búsqueda de qué se dice y cómo sobre cualquier fenómeno o personaje literario. Sobre estos análisis y sobre la temática literaria y su relevancia en la cultura entendida como un todo, vertebramos la vinculación de nuestras asignaturas.

La colaboración docente conlleva la aplicación de metodologías activas y participativas para el estudiante y la adecuación de los sistemas de evaluación a estas metodologías. De esta manera, se potencia el desarrollo de competencias de utilidad social por los alumnos a la hora de formar parte del mercado del trabajo. La metodología docente adecuada por sí misma y por aglutinadora de otros métodos docentes complementarios es la del seminario. Son los alumnos los encargados de prepararlo y son sus participantes principales, junto con alguna figura literaria o crítico de relevancia como invitado. El seminario propicia la interrelación entre el alumnado, y no sólo de un mismo curso, en el caso propuesto.

De los distintos tipos de métodos didácticos, siguiendo a Miguel Díaz (Miguel, 2005, p. 37), apostamos por el predominio del seminario en combinación con la lección tradicional, método de caso, grupo pequeño de trabajo, metodología de aprendizaje cooperativo y resolución de problemas. Nuestra metodología está centrada en los enfoques denominados por Miguel Díaz de la socialización didáctica y enfoque globalizado (Miguel, 2005, p. 38-39). Hablamos de metodologías docentes activas, coincidiendo con Rodríguez Jaume en las que se produce un cambio de papel entre profesor y alumno (Rodríguez Jaume, 2006).

Al escoger como un método de enseñanza el aprendizaje cooperativo - y siendo nosotras un equipo también cooperativo - , según Felder y Brent, hemos de intentar 
En concreto, la planificación de nuestras asignaturas de Literatura y medios de comunicación, Inglés y Redacción para los medios III conlleva el establecimiento de las siguientes metodologías, vinculadas a las competencias y resultados de aprendizaje descritos anteriormente:

El seminario nos permite desarrollar las competencias fijadas en el Grado de Periodismo, puesto que conlleva la interacción entre práctica y teoría logrando que el alumno profundice en los contenidos propuestos para cada asignatura. Nuestro acercamiento a hechos reales considerados como informativos desde la literatura y sus avatares, y desde distintas disciplinas, precisa de un método como el seminario con el objeto de conseguir una formación integral y humanística, así como adecuarnos al perfil profesional del periodista. Cabe recordar las funciones del seminario que exponen autores como Riera, Giné y Castelló:

1. Orientación y seguimiento del estudiante en su proceso de aprendizaje autónomo y autorregulado. 2. Vivencia de un clima de trabajo y de estímulo intelectual cooperativo. 3. Aportación al estudiante de la experiencia intelectual y profesional del profesor-tutor. 4. Fomento de la capacidad de síntesis interdisciplinaria. 5. Promoción de la transferencia de los conocimientos a la práctica profesional. 6. Promoción de la adquisición de estrategias y técnicas para el trabajo autónomo del estudiante en función de los intereses personales y grupales. 7. Fomento de las capacidades de diálogo, argumentación, retórica y de contraste de opiniones. 8. Promoción de las habilidades y actitudes necesarias para el trabajo en equipos con intereses interdisciplinarios diversos. 9. Desarrollo de la capacidad de evaluación y autoevaluación del propio proceso de aprendizaje. (Riera; Giné, \& Castelló, 2003)

El método del seminario se fundamenta en un trabajo conjunto entre alumnos y docentes. El papel de los estudiantes pasa por una implicación activa en su aprendizaje que, paulatinamente, facilita un acceso autónomo al conocimiento. Con el paso de un curso a otro, "los alumnos acceden a niveles más elevados en la responsabilidad de la dinámica del seminario y en la organización de las actividades de enseñanza-aprendizaje del mismo" (Riera, et al., 2003, p. 250-251). Precisamente, nuestra propuesta consiste en la puesta en marcha de un seminario conjunto (entre primero, segundo y tercer curso) y anual sobre Literatura y periodismo al final de nuestras asignaturas.

Los alumnos de primer curso del grado de periodismo y en la asignatura de Literatura y medios de comunicación se encargan de liderar una mesa redonda en la que distintos portavoces de equipos de trabaio contextualicen la vida v obra de figuras 
Son los alumnos de segundo, con la asignatura de Inglés, y estableciendo un mayor grado de dificultad, los que analicen y presenten el tratamiento de los autores en los tabloides y la prensa seria anglosajona, siguiendo las principales cabeceras internacionales, desde el método de análisis configurado en nuestras investigaciones.

Sus portavoces presentarán en inglés sus descubrimientos. Ya en tercero, los estudiantes centran su atención en la prensa española, deteniéndose en los géneros de interpretación y de opinión periodísticos, propios del último nivel de redacción establecido con la asignatura Redacción para los medios III. Entre todos se contribuye a la configuración de un perfil de los autores literarios elegidos. Y los alumnos son conscientes del papel del periodismo en la difusión del perfil mencionado. Como broche del seminario, los alumnos conforman una mesa redonda contando con un literato reconocido como invitado, con el que discuten sus descubrimientos.

En el rol docente, se requiere una visión amplia de la profesión, de la interdisciplinariedad y de la dinámica de grupos que invite a la reflexión del alumno y que propicia "un clima de participación, de respeto, de acogida y de integración de todos los miembros del grupo y de sus aportaciones" (Riera, et al., 2003, pp. 251). Y creemos que esta actividad lo permite claramente.

Para la consecución exitosa del método del seminario, un método sin duda aglutinador de otros tantos, requerimos también la utilización del método de aprendizaje cooperativo, la lección tradicional, el método de caso, la resolución de problemas y el grupo pequeño de trabajo.

En el caso del método de aprendizaje cooperativo, y siguiendo a Monereo, prima la interacción entre compañeros como "fuente de conocimiento". Este autor afirma que: "el trabajo cooperativo puede ser una alternativa muy eficaz para enseñar a los alumnos no sólo nuevas formas de gestionar socialmente el conocimiento, sino también nuevos conocimientos" (Monereo, 2003, p. 27-28). Requiere de discusiones grupales, basadas en lecturas previas, reflexión, conexión con otros asuntos relacionados, visión crítica, etc., en torno al planteamiento de un problema o de una pregunta por parte del docente.

Y que luego se plasman en la presentación de síntesis en el aula. Este tipo de método colaborativo "ayuda a los alumnos a prepararse para trabajar con otros, para liderar grupos, para tomar decisiones", en palabras de Marcelo y Estebaranz quienes, además, vinculan el trabajo cooperativo como "un complemento de la clase magistral" (Marcelo \& Estebaranz, 2003, p. 22). 
Para la consecución del seminario de periodismo y literatura propuesto, este método está obvia y previamente presente en nuestra planificación, teniendo en cuenta las características de cada asignatura. La llamada lección tradicional sirve como punto de partida para integrar después a los estudiantes en grupos pequeños de trabajo que preparan el seminario utilizando también el método de caso y de resolución de problemas. Todos estos métodos presentados ayudan tanto al desarrollo de competencias como al logro de distintos resultados de aprendizaje.

Esperamos que la evaluación para el aprendizaje de nuestros alumnos de la que hablan Águeda Benito (Águeda Benito, 2009, p. 19-21) o Mario de Miguel (Miguel, 2005, p. 42-48) resulte positiva, puesto que, en consonancia con lo ya escrito en líneas anteriores y siguiendo a Mateos y Peñalba (Mateos \& Peñalba, 2003, p. 91) queremos que aprendan a entender y aprovechar textos literarios y periodísticos "adoptando un enfoque profundo" y "planteándoles tareas o problemas abiertos que requieran un uso productivo, y no meramente reproductivo, de la información adquirida".

Como profesores universitarios nuestra enseñanza está centrada, siguiendo a Gairín en "autoestudio-tutoría-trabajo-evaluación-satisfacción" (Gairín, 2003, p. 129). Sintetiza Isabel Solé que como docentes hemos de:

En primer lugar, preguntar y preguntarnos qué queremos que sepan, sepan hacer y sean los futuros profesionales cuya formación inicial está en nuestras manos (...). En segundo lugar, habría que preguntarse cómo estos objetivos pueden lograrse a lo largo de una oferta formativa que se prolonga durante varios años y que incluye diversas modalidades (Solé, 2003).

Y para concluir, seguimos coincidiendo con Solé:

Sería fundamental aceptar que cada una de las asignaturas que se imparte, por importante que sea y a cada uno se lo parezca, no constituye un compartimento estanco, sino un ámbito cuyo conocimiento debería proporcionar instrumentos conceptuales, estrategias y técnicas y contribuir a la formación de actitudes y valores necesarios para comprender e intervenir en la parcela de realidad que se constituye como ámbito profesional o de conocimiento. (Solé, 2003)

\section{CONCLUSIONES}

A modo de conclusión, el EEES da cabida a metodologías interdisciplinares y

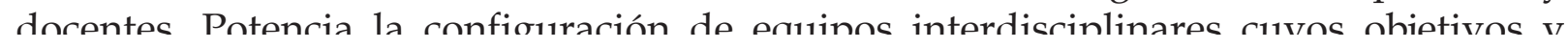


De ambas metodologías, interdisciplinares y docentes, surgen planteamientos metodológicos globalizadores cuyos materiales de aprendizaje se secuencian y asemejan en gran medida a la realidad profesional y cotidiana de la que formarán parte los alumnos egresados. La toma de conciencia de la conexión y la complementariedad se da no sólo en el alumno sino también en los profesores.

El encuentro entre disciplinas, fundamental para el avance de la propia ciencia, permite esa educación global del alumno que le ayude a interrelacionar todos los conocimientos adquiridos a su paso por la universidad y a integrarse en el mercado del trabajo.

Serán las metodologías docentes activas e interdisciplinares una baza relevante. Y el seminario como método idóneo por sí mismo y como aglutinador de otros contribuye a la formación integral y global del alumno de periodismo, cuya profesión se basa esencialmente en saber comunicar.

\section{REFERENCIAS}

Benito, A. (2009). El proceso de evaluación como herramienta activadora del aprendizaje del alumno. Estrategias de innovación en el nuevo proceso de evaluación de aprendizaje. Valladolid: Servicio de Publicaciones UEMC.

Gairín, J. (2003). El profesor universitario en el siglo XXI. En La universidad ante la nueva cultura educativa. Enseñar y aprender para la autonomía. Madrid: Síntesis.

López Pan, F. (2007). La adecuación de los programas docentes a las competencias profesionales en las titulaciones del campo de las Ciencias de la Información: una formación de transición hacia el mercado laboral. En Inserción laboral y Espacio Europeo de Educación Superior: Aplicación de las Ciencias de la Información. Valladolid: Servicio de Publicaciones UEMC.

Marcelo García, C. \& Estebaranz García, A. (2003). Marco general de investigación sobre la enseñanza en la universidad. En Enseñanza y aprendizaje en la educación superior. Barcelona: Octaedro-EUB.

Martí, E. (2003). Conclusiones: un currículo para desarrollar la autonomía del estudiante. En La universidad ante la nueva cultura educativa. Madrid: Síntesis.

Martín-Izard, J. F.; Ausín, T.; Vivar-Quintana, A. M.; Revilla, I. \& Rodríguez, M. A. (2008). Un proyecto de innovación educativa basado en la creación de equipos 
Mateos, M \& Peñalba, G. (2003). Aprendizaje a partir del texto científico en la universidad. En La universidad ante la nueva cultura educativa. Enseñar y aprender para la autonomía. Madrid: Síntesis.

Miguel Díaz, M. de. (2005). Modalidades de enseñanza en el desarrollo de competencias. Oviedo: Ediciones Universidad de Oviedo.

Monereo, C. \& Pozo, J. I. (2003). La cultura educativa en la universidad: Nuevos retos para profesores y alumnos. En La universidad ante la nueva cultura educativa. Enseñar y aprender para la autonomía. Madrid: Síntesis.

Riera, J., Giné, C. \& Castelló, M. (2003). El seminario en la universidad. Un espacio para la reflexión sobre el aprendizaje y para la formación. En La universidad ante la nueva cultura educativa. Enseñar y aprender para la autonomía. Madrid: Síntesis.

Rodríguez Jaume, Ma J. (2006). Espacio Europeo de Educación Superior y Metodologías docentes activas. Dossier de trabajo. Alicante: Universidad de Alicante.

Solé, I. (2003). Conclusiones: el profesor universitario en el siglo XXI. En La universidad ante la nueva cultura educativa. Enseñar y aprender para la autonomía. Madrid: Síntesis.

Sotelo, J. \& Marco, J. C. (2008). Una reflexión sobre la metodología docente sobre la enseñanza del Periodismo en el marco del EEES. En El nuevo perfil del profesor universitario en el EEES: claves para la renovación metodológica. Valladolid: Servicio de Publicaciones UEMC.

\section{Rosa María Arráez Betancort}

Doctora en Periodismo por la Universidad Complutense de Madrid y Máster en Periodismo y comunicación científica por la UNED. Ha realizado estancias de investigación en la Universidad de Texas (Estados Unidos) y en el Tecnológico de Monterrey (México). En su faceta divulgadora científica destacan las publicaciones Ciencia para informadores: Nociones básicas de periodismo científico, Medios de comunicación y desarrollo sociocultural, Aceleración y postmodernidad en el microrrelato o La fotografía en la información científica de la prensa generalista española, etc. Y la organización de cursos anuales sobre periodismo científico. Ha sido columnista quincenal de El Mundo de Castilla y León con la columna Vía de Escape durante 4 años. 


\section{Elvira Jensen Casado}

Doctora en Filología Inglesa por la universidad de Salamanca imparte clases de English for Specific Purposes en los grados de Periodismo, Comunicación Audiovisual y ADE en la Universidad Europea Miguel de Cervantes, anteriormente lo ha hecho en la Universidad de Salamanca y en la Universidad Católica San Antonio Murcia. Así mismo ha participado en el programa Minerva (ODL and ICT in Education) de la Unión europea: An educational dimension of conflict resolution through cultural production.

\section{Carolina Pascual Pérez}

Licenciada de grado en Filología Hispánica por la universidad de Valladolid y ABD en literatura española contemporánea por la Universidad de Ohio State (Columbus, EE.UU.). Imparte clases de Lengua española y Literatura y medios de comunicación en la Universidad Europea Miguel de Cervantes. Sus principales líneas de investigación están relacionadas con el análisis semiótico de símbolos en el teatro, cine, literatura, etc., así como el estudio de la literatura española y portuguesa del siglo XIX. Actualmente forma parte del grupo de investigación que lleva a cabo el proyecto de I+D+i (2009-2011) financiado por la Consejería de Educación de CyL. Cuenta con diferentes publicaciones y participaciones en congresos nacionales e internacionales. 